\title{
Ethnic Differences in Intracranial Artery Tortuosity: A Possible Reason for Different Locations of Cerebral Atherosclerosis
}

\author{
Bum Joon Kim, ${ }^{a}$ Kyung Mi Lee, ${ }^{\mathrm{b}}$ Sung-Ho Lee, ${ }^{\mathrm{c}}$ Hyug-Gi Kim, ${ }^{\mathrm{b}}$ Eui Jong Kim, ${ }^{\mathrm{b}}$ Sung Hyuk Heo, ${ }^{\mathrm{a}}$ \\ Dae-il Chang, ${ }^{\text {a Jong S. Kim }}{ }^{\mathrm{d}}$
}

\begin{abstract}
aDepartment of Neurology, Kyung Hee University Hospital, Kyung Hee University School of Medicine, Seoul, Korea 'Department of Radiology, Kyung Hee University Hospital, Kyung Hee University School of Medicine, Seoul, Korea 'Department of Neurosurgery, Kyung Hee University Hospital, Kyung Hee University School of Medicine, Seoul, Korea ${ }^{\mathrm{d} D e p a r t m e n t}$ of Neurology, Asan Medical Center, University of Ulsan College of Medicine, Seoul, Korea
\end{abstract}

\section{Dear Sir:}

It remains unclear why intracranial artery atherosclerosis (ICAS) is more common in Asians than in Caucasians. ${ }^{1,2}$ Intracranial arterial tortuosity is associated with atherosclerotic plaque, ${ }^{3}$ and is greater in stroke patients with ICAS than in those without. ${ }^{4}$ Therefore, we hypothesized that arterial tortuosity may be greater in Asians than in Caucasians, and compared the tortuosity between age-/sexmatched Koreans and Caucasians.

Among 339 "white-ethnicity" subjects who visited the Health Promotion Center at Kyung Hee University Medical Center from 2015 to 2016, we enrolled 76 without a history of stroke or cerebral artery steno-occlusion and performed magnetic resonance imaging (MRI) and magnetic resonance angiography (MRA). Controls included 76 age- and sexmatched Koreans from the same center. This study was approved by the local Institutional Review Board.

The source image of time-of-flight MRA obtained from a 3-T MRI system was used for measurement. The tortuosity of middle cerebral arteries (MCAs, M1 portion) and the basilar artery (BA) was measured semi-automatically using a commercial image analysis program (TeraRecon, TeraRecon Inc., San Mateo, CA, USA). Tortuosity was defined as the ratio between the actual length and the minimal straight length between the endpoints of each artery. ${ }^{4}$ The intracranial artery tortuosity index (ICATI) was defined as the mean of the tortuosity indexes [(actual length/ straight length-1)×100] of the right and left MCAs and BA.

Arterial tortuosity was significantly greater in the left MCA and $B A$, and marginally greater in the right MCA in Koreans than in whites (Table 1). The ICATI was higher in Koreans than in whites. Multiple logistic regression analysis including age, sex, risk factors, and ethnicity showed that high ICATI was independently associated with Korean ethnicity (odds ratio, 1.10; 95\% confidence interval $[\mathrm{Cl}], 1.03$ to $1.17 ; P=0.002)$. ICATI was independently associated with age $(\beta=0.214 ; 95 \%$ $\mathrm{Cl}, 0.039$ to $0.388 ; P=0.017)$ and hypertension $(\beta=4.635$; $95 \% \mathrm{Cl}, 1.255$ to $8.014 ; P=0.008)$ in Koreans, but not in whites. ICATI was significantly higher in hypertensive Koreans than in non-hypertensive Koreans ( $16.5 \pm 9.9$ vs. $10.8 \pm 4.5$, $P=0.001)$, whereas there was no such difference in whites (Figure 1A). ICATI was significantly higher in Koreans than in whites among hypertensive subjects $(P=0.002)$, whereas there was no such difference in non-hypertensive subjects. ICATI increased with age in Koreans (correlation efficiency $=0.340$, $P=0.003$ ), but not in whites (Figure 1B).

We found that intracranial arterial tortuosity was greater in Koreans than in whites, and that Korean ethnicity was independently associated with tortuosity. However, this ethnic difference was not apparent among normotensive subjects. Intracranial arterial tortuosity was greater in hypertensive Koreans than in normotensive Koreans; this pattern was not observed in whites. Furthermore, the association of arterial tortuosity with old age and hypertension was ob- 
Table 1. Comparison between Koreans and whites

\begin{tabular}{lccc}
\hline Variable & Koreans $(\mathrm{n}=76)$ & Whites $(\mathrm{n}=76)$ & $P$ \\
\hline Age $(\mathrm{yr})$ & $56.1 \pm 9.6$ & $56.1 \pm 9.6$ & $78(51.3)$ \\
Male sex & $78(51.3)$ & $33(43.4)$ & 0.870 \\
Hypertension & $32(42.1)$ & $8(10.5)$ & 0.797 \\
Diabetes & $9(11.8)$ & $15(19.7)$ & 0.691 \\
Hyperlipidemia & $17(22.4)$ & $26(34.2)$ & 0.905 \\
Current smoking & $26(34.2)$ & $9.6 \pm 5.2$ & 0.001 \\
Intracranial artery tortuosity index & $13.2 \pm 7.8$ & $10.4 \pm 9.9$ & 0.227 \\
Right MCA tortuosity index & $12.3 \pm 9.1$ & $10.3 \pm 7.3$ & 0.001 \\
Intracranial artery tortuosity index & $13.6 \pm 16.1$ & $10.3 \pm 7.3$ & 0.023 \\
BA tortuosity index & $13.7 \pm 10.7$ & \\
\hline
\end{tabular}

Values are presented as mean \pm standard deviation or number (\%). Tortuosity index: [(actual length/straight length)-1]×100. The intracranial artery tortuosity index is the mean of the tortuosity of the right and left MCA and BA.

$M C A$, middle cerebral artery; BA, basilar artery.

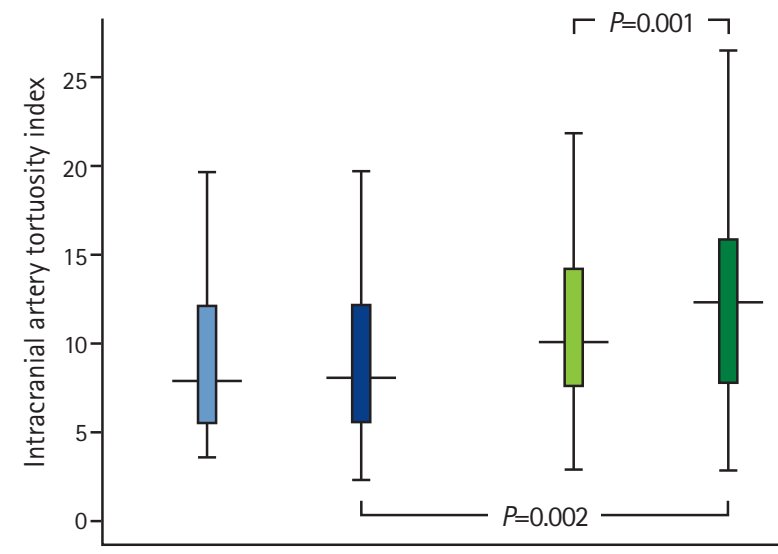

$\square$ Nonhypertensive-Whites $\square$ Nonhypertensive-Koreans $\square$ Hypertensive-Whites

口Hypertensive-Koreans

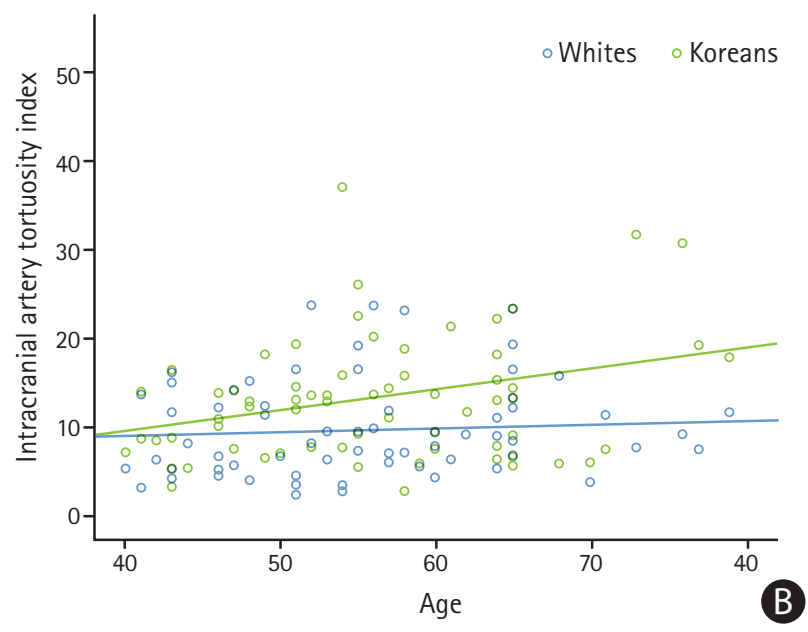

Figure 1. Intracranial artery tortuosity index in subjects with and without hypertension (A) and according to age (B) in different ethnicities (green: Koreans; blue: whites).

served in Koreans, but not in whites. Thus, intracranial arteries may be more vulnerably influenced by hemodynamic stress induced by age and hypertension in Koreans than in whites. The greater intracranial arterial tortuosity in Koreans with vascular risk factors may in part explain the higher prevalence of ICAS in Asians than in whites. Further studies are required to prove these preliminary findings.

\section{References}

1. Kim JS, Kim YJ, Ahn SH, Kim BJ. Location of cerebral atherosclerosis: why is there a difference between east and west? Int J Stroke 2018;13:35-46.

2. Yang WJ, Wong KS, Chen XY. Intracranial atherosclerosis: from microscopy to high-resolution magnetic resonance imaging. J Stroke 2017;19:249-260.

3. Kim BJ, Yoon Y, Lee DH, Kang DW, Kwon SU, Kim JS. The shape of middle cerebral artery and plaque location: highresolution MRI finding. Int J Stroke 2015;10:856-860.

4. Kim BJ, Kim SM, Kang DW, Kwon SU, Suh DC, Kim JS. Vascular tortuosity may be related to intracranial artery atherosclerosis. Int J Stroke 2015;10:1081-1086.

Correspondence: Jong S. Kim

Department of Neurology, Asan Medical Center, University of Ulsan College of Medicine, 88 Olympic-ro 43-gil, Songpa-gu, Seoul 05505, Korea Tel: $+82-2-3010-3442$

Fax: +82-2-474-4691

E-mail: jongskim@amc.seoul.kr

Received: December 17, 2017

Revised: January 10, 2018

Accepted: January 18, 2018

This work was supported by a grant from the Ministry of Health, Welfare and Family Affairs, Republic of Korea (HI14C1985).

The author has no financial conflicts of interest. 